\title{
Heavy ion beam acceleration in the first three cryomodules at the Facility for Rare Isotope Beams at Michigan State University
}

\author{
P. N. Ostroumov, ${ }^{*}$ S. Cogan, K. Fukushima, S. Lidia, T. Maruta, \\ A. S. Plastun, J. Wei, J. Wong, T. Yoshimoto, and Q. Zhao \\ Facility for Rare Isotope Beams, Michigan State University, East Lansing, Michigan 48824, USA
}

(Received 10 February 2019; published 5 April 2019)

\begin{abstract}
The Facility for Rare Isotope Beams (FRIB) being constructed at Michigan State University [J. Wei et al., The FRIB superconducting linac—status and plans, LINAC'16, Lansing, MI, p. 1, http://accelconf .web.cern.ch/AccelConf/linac2016/papers/mo1a01.pdf] is based on a cw superconducting linear accelerator which is designed to deliver unprecedented $400 \mathrm{~kW}$ heavy ion beam power to the fragmentation target. The installation of the accelerator equipment is approaching completion and multistage beam commissioning activities started in the summer of 2017 with expected completion in 2021. A roomtemperature test electron cyclotron resonance ion source, ARTEMIS, provided argon and krypton beams for the commissioning of the low energy beam transport, a radio frequency quadrupole (RFQ), the medium energy beam transport (MEBT) and the first three accelerating cryomodules. The commissioning of the first linac segment (LS1), composed of 15 cryomodules, is planned in the spring of 2019. This paper describes the first results of experimental beam dynamics studies in the LEBT, RFQ, MEBT and the first three cryomodules with comparison to the numerical simulations.
\end{abstract}

DOI: 10.1103/PhysRevAccelBeams.22.040101

\section{INTRODUCTION}

Argon and krypton ion beams were transported to the radio frequency quadrupole (RFQ) and successfully accelerated in the RFQ in September of 2017 [1]. After that, there was a ten-month period of intermittent operation of the front end for beam physics studies. The next stage of the FRIB linac commissioning took place in the summer of 2018 and included acceleration of argon and krypton beams in the first three cryomodules, which contain $12 \beta_{\mathrm{OPT}}=$ 0.041 superconducting (SC) cavities and six SC solenoids. The hardware layout for this summer 2018 commissioning stage is shown in Fig. 1. The temporary diagnostics station (D-station), installed after the third cryomodule, included ac-coupled beam current monitors (BCMs), a Faraday cup (FC), beam position monitors (BPMs), halo monitor rings, a profile monitor $(\mathrm{PM})$ and a silicon detector $(\mathrm{SiD})$. The design energy for both the argon and krypton beams is $1.46 \mathrm{MeV} / \mathrm{u}$ in this section of the linac. The primary goals during the commissioning were: (i) confirmation of the accelerator design and required functionality; (ii) detailed study of accelerated beam parameters; (iii) demonstration of the highest beam energy (with available accelerating

\footnotetext{
*ostroumov@frib.msu.edu
}

Published by the American Physical Society under the terms of the Creative Commons Attribution 4.0 International license. Further distribution of this work must maintain attribution to the author(s) and the published article's title, journal citation, and DOI. gradients) in the first three cryomodules; and (iv) demonstration of high-power equivalent beam in a pulsed mode.

For efficient use of the beam time, a set of on-line physics applications has been developed to support: (i) low energy beam transport (LEBT) tuning; (ii) optimal setting of the multiharmonic buncher (MHB) phases and fields; (iii) beam central trajectory correction in LEBT, medium energy beam transport (MEBT) and cryomodules; (iv) quadrupole or solenoid scan for profile measurements and evaluation of rms emittance; (v) phase scan of $\mathrm{rf}$ cavities; (vi) BPM-based TOF measurements to determine the absolute beam energy; and (vii) data processing from the silicon detector.

This paper consists of three sections describing beam dynamics studies in (1) the LEBT, (2) the RFQ and MEBT, and (3) the first three cryomodules.

\section{LOW ENERGY BEAM TRANSPORT}

The LEBT includes two ECR ion sources: the room temperature ARTEMIS-B [2] (ECRIS-1) and the superconducting ion source with similar parameters as VENUS [3] (ECRIS-2). The FRIB linac commissioning was performed using ion beams extracted from the ARTEMIS-B. As described in previous publications [4], the ARTEMIS-B is installed on high voltage decks to provide extraction and acceleration of all ion species generated in the ECR. The mass and charge selection takes place between two $90^{\circ}$ dipole magnets, where the dispersion reaches its highest value. This LEBT allows us to select and transport 


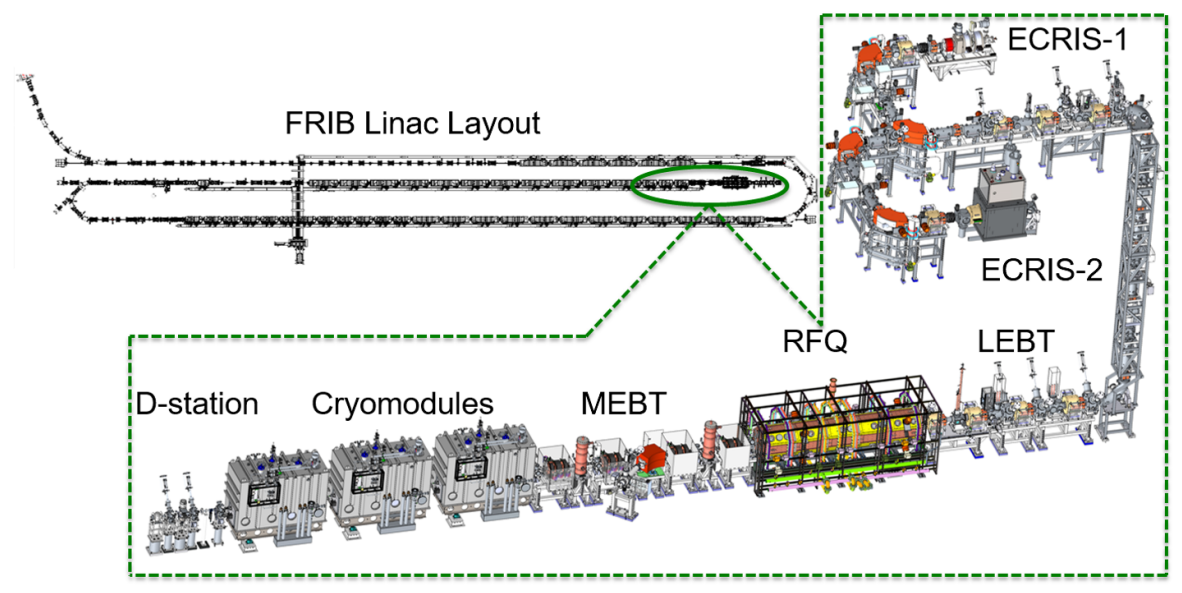

FIG. 1. FRIB layout including the front end, the first three cryomodules and commissioning diagnostics station (D-station). The future superconducting Electron Cyclotron Resonance Ion Source (ECRIS-2) is also shown.

two-charge-state heavy ion beams simultaneously for further acceleration and delivery of higher beam power to the FRIB fragmentation target. This feature will be used to reach $400 \mathrm{~kW}$ beam power on the target. The LEBT is equipped with a large amount of various beam diagnostics devices, as shown in Fig. 2. The beam current measurements were performed primarily with Faraday cups. The FCs were biased to recapture the secondary electrons and provide accurate measurements. The current measurement accuracy of FCs is $1 \%$, determined mainly by the calibration procedure. In the summer of 2018 we began to use an electrostatic chopper (see Fig. 2) to produce a pulsed beam structure which enabled BCMs for beam current measurements. The BCMs were calibrated to $1 \%$ accuracy. The FCs have sensitivity down to $\sim 10 \mathrm{pA}$ while the BCMs are noise floor limited to $\sim 1 \mu \mathrm{A}$ on fast timescale $(1 \mathrm{MHz})$. The beam current from the ion source is prone to fluctuations of $\pm 5 \%$ within the time frame of $1-3$ msec.

The LEBT is a rather complicated optical system consisting of solenoids, bending magnets, electrostatic quadrupoles and dipoles. The performance of the FRIB front end (FE) per project specifications was successfully

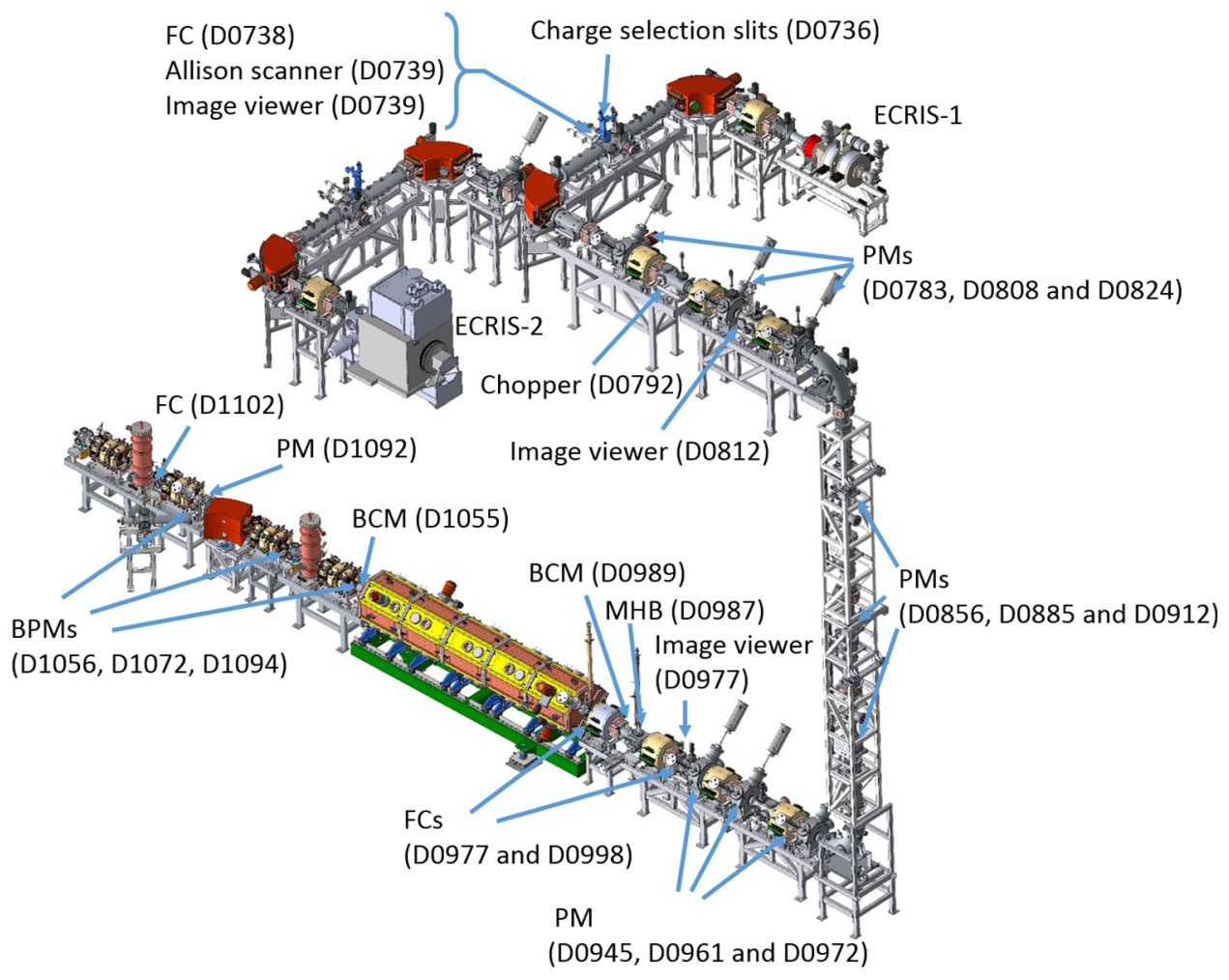

FIG. 2. Layout of the LEBT with the location of beam instrumentation. Each device has a decimeter number showing the location along the FRIB beam line. The RFQ and MEBT are also shown. 
demonstrated shortly after the first beam commissioning started. The results were reported elsewhere [2]. After the project goals were demonstrated, we had an opportunity for extensive beam physics studies in the FRIB FE to accomplish the following tasks: (i) evaluation of the beam rms parameters and emittance from measured data; (ii) beam optics tuning to create a small horizontal beam size in the charge selection slits; (iii) beam based alignment of beam diagnostics devices such as image viewers, Allison scanner and profile monitors; (iv) minimization of transverse emittance growth due to $\mathrm{X}-\mathrm{Y}$ coupling of nonaxially symmetric beam; (v) beam central trajectory correction along the LEBT; (vi) beam matching into the RFQ transverse acceptance; (vi) transport of dual-charge-state krypton beam and matching to the RFQ.

All studies were performed with argon beam, except the last item in the above-mentioned list. The beam optics devices such as solenoids, quadrupoles and dipoles were aligned, with high accuracy, to $\pm 100 \mu \mathrm{m}$. A beam-based alignment procedure was applied to the charge selection slits, image viewers, Allison emittance scanner and profile monitors. This technique includes beam centering in an upstream focusing device and alignment of the diagnostic devices with respect to the focusing device. In these experiments, the ${ }^{40} \mathrm{Ar}^{9+}$ beam intensity was within the range from 10 to $110 \mu \mathrm{A}$.

In the LEBT, the beam second moments can be written as a $\sigma$ matrix:

$$
\begin{aligned}
\sigma & =\left(\begin{array}{cccc}
\langle x x\rangle & \left\langle x x^{\prime}\right\rangle & \langle x y\rangle & \left\langle x y^{\prime}\right\rangle \\
\left\langle x x^{\prime}\right\rangle & \left\langle x^{\prime} x^{\prime}\right\rangle & \left\langle x^{\prime} y\right\rangle & \left\langle x^{\prime} y^{\prime}\right\rangle \\
\langle x y\rangle & \left\langle x^{\prime} y\right\rangle & \langle y y\rangle & \left\langle y y^{\prime}\right\rangle \\
\left\langle x y^{\prime}\right\rangle & \left\langle x^{\prime} y^{\prime}\right\rangle & \left\langle y y^{\prime}\right\rangle & \left\langle y^{\prime} y^{\prime}\right\rangle
\end{array}\right) \\
& =\left(\begin{array}{llll}
\sigma_{11} & \sigma_{12} & \sigma_{13} & \sigma_{14} \\
\sigma_{21} & \sigma_{22} & \sigma_{23} & \sigma_{24} \\
\sigma_{31} & \sigma_{32} & \sigma_{33} & \sigma_{34} \\
\sigma_{41} & \sigma_{42} & \sigma_{43} & \sigma_{44}
\end{array}\right)
\end{aligned}
$$

The $\sigma$ matrix is symmetric (e.g., $x x^{\prime}=x^{\prime} x, x y=y x$, etc.) and the beam can be fully characterized with ten rms parameters: $\sigma_{11}, \sigma_{12}, \sigma_{13}, \sigma_{14}, \sigma_{22}, \sigma_{23}, \sigma_{24}, \sigma_{33}, \sigma_{34}, \sigma_{44}$.

The TRACK code [5] was utilized for multiparticle simulations of multicomponent ion beams in the 3D electromagnetic fields. This code was also utilized for fitting of beam parameters from the measured data and for optimal settings of beam line elements. The fitting and optimization capability of the simulation code, TRACK, was enhanced using a PYTHON environment. We found very good agreement of the beam rms parameters simulated by TRACK multiparticle code and FLAME "envelope" code [6] for single component ion beams. In most cases, the fitting and optimization of single-component ion beam rms parameters and beam optics settings were performed with the fast FLAME code. For the TRACK simulations, we generated initial distribution in the 4D phase space at the location of the ECRIS extraction electrode, which is inside the solenoid. For a given $\sigma$ matrix, the same 4D Gaussian distribution was generated for all components of different ions at different charge states. The total current of the multicomponent ion beam is equal to the measured drain current of the ECRIS. The intensity of an individual ion component was determined as a result of mass and charge state analysis, downstream of the $90^{\circ}$ dipole magnet. Several methods were applied to reconstruct the beam $\sigma$ matrix at the ECR extraction electrode. For low intensity beams, below $\sim 25 \mu \mathrm{A}$, a beam image viewer, D0739 in Fig. 2, was utilized for the measurements of the beam density distribution in the $\mathrm{XY}$ plane; to calculate second moments $\sigma_{11}, \sigma_{33}, \sigma_{13}$. These measurements were performed multiple times by varying the field of the electrostatic quadrupoles upstream of the viewer [7]. These datasets were used to find the beam $\sigma$ matrix at the ECR extraction electrodes by rms fitting with the TRACK code. The ten unknown parameters of the $\sigma$ matrix were found with an optimization scheme based on NelderMead's method [8] to reproduce the measured rms beam sizes, $\sqrt{\sigma_{11}}, \sqrt{\sigma_{33}}$ and coupling coefficient, $\chi=\frac{\sigma_{13}}{\sqrt{\sigma_{33} \sigma_{11}}}$. The $25 \mu \mathrm{A}$ argon beam $\sigma$ matrix at the ECRIS extraction electrode was calculated to be (beam coordinates are measured in $[\mathrm{mm}]$ and $[\mathrm{mrad}])$

$$
\sigma=\left(\begin{array}{cccc}
3.07 & -9.61 & -1.05 & 10.1 \\
-9.61 & 274 & -6.00 & -84.6 \\
-1.05 & -6.00 & 0.90 & -3.13 \\
10.1 & -84.6 & -3.13 & 165
\end{array}\right)
$$

The beam is not axially symmetric with the coupling $\chi=-0.63$. In addition, the $\mathrm{X}$ and $\mathrm{Y}$ emittances are different.

The projections of the $4 \mathrm{D}$ beam phase space to the $X X^{\prime}$ and $\mathrm{YY}^{\prime}$ planes can be directly measured with the Allison scanner, after the charge and mass selection. Typical phase space plots obtained with the Allison scanner are shown in Fig. 3. However, these measurements do not provide insight to the coupling terms in the $\sigma$ matrix. We also evaluated the beam $\sigma$ matrix using nine independent profile measurements along the LEBT. Figure 4 shows the rms envelopes for $50 \mu \mathrm{A}$ argon beam along the LEBT, with the initial beam $\sigma$ matrix fitted to match the measured beam rms sizes along the LEBT. Ten elements of the beam $\sigma$ matrix at the location of charge stripper are shown in Table I, evaluated with two different methods.

At lower beam intensities, below $\sim 25 \mu \mathrm{A}$, image viewers can be used to extract beam density distribution in the XY plane. Figure 5 shows the measured beam images along the LEBT, together with simulated beam images in the same 

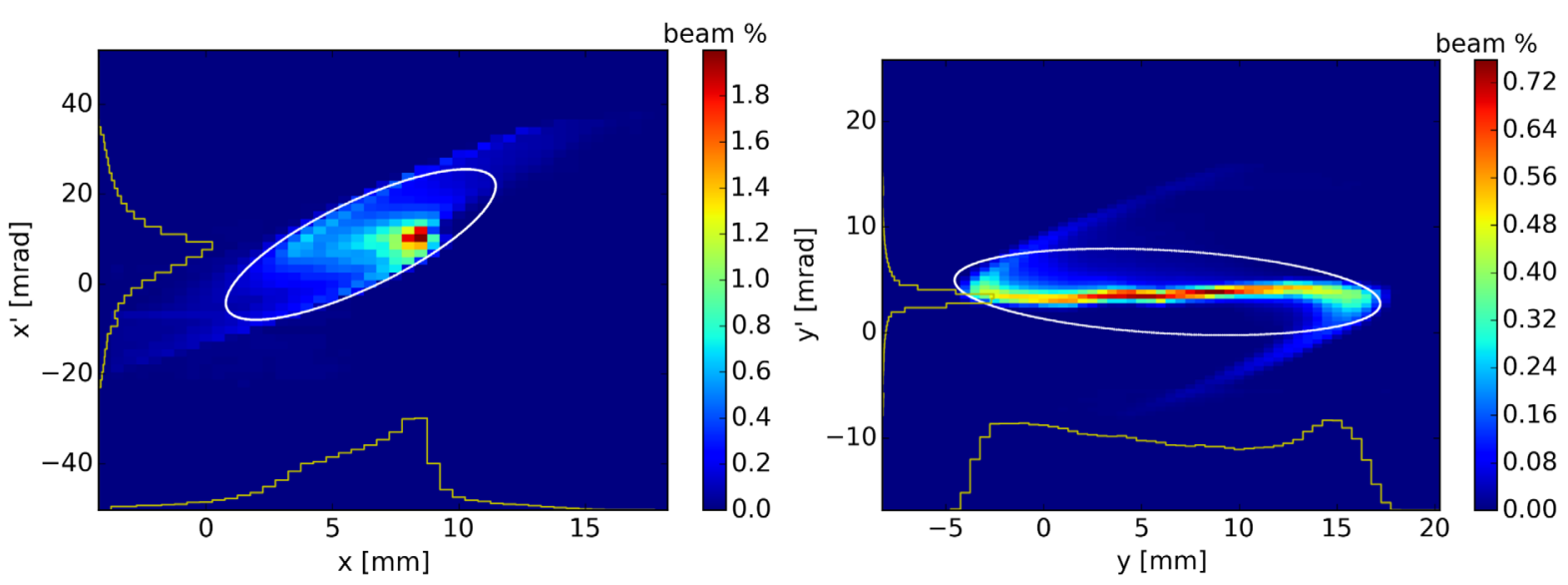

FIG. 3. Typical beam emittances measured with the Allison scanner.

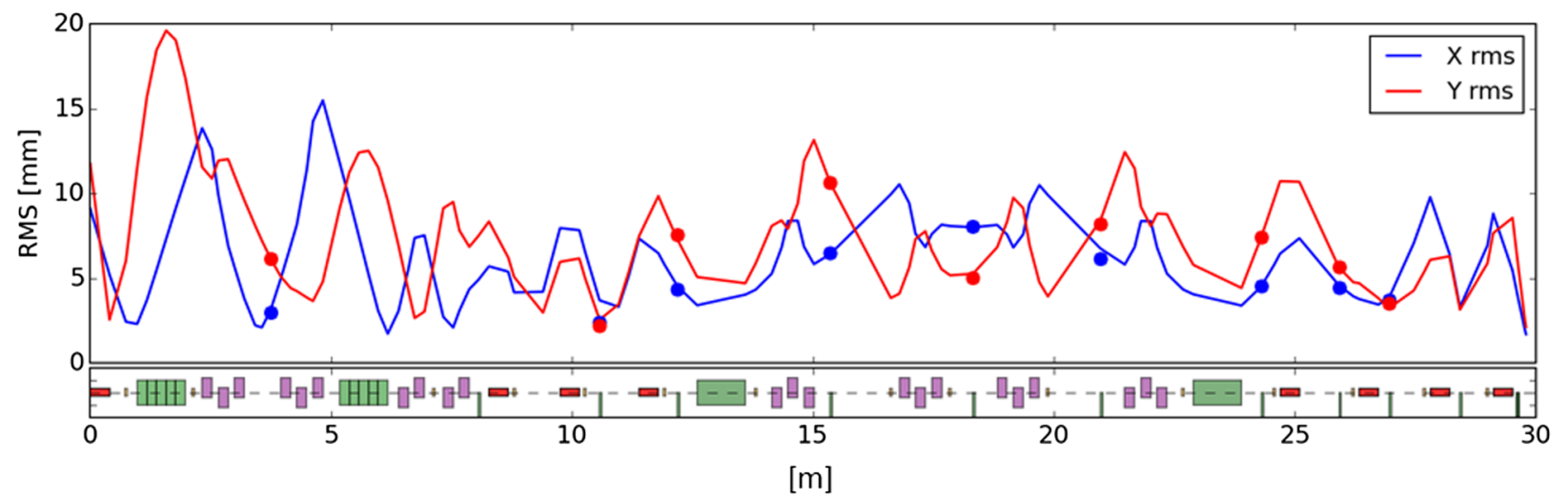

FIG. 4. Argon beam rms envelopes (solid lines) in the LEBT with the beam initial parameters fitted to match the measured (dots) beam rms sizes along the LEBT.

TABLE I. Elements of the argon beam $\sigma$ matrix at the location of the charge selector. $12 \mathrm{keV} / \mathrm{u}$ argon beam current is $50 \mu \mathrm{A}$.

\begin{tabular}{|c|c|c|c|}
\hline Parameter & $\begin{array}{c}\text { Allison } \\
\text { scanner D0739 }\end{array}$ & $\begin{array}{l}\text { Quad } \\
\text { scan }\end{array}$ & $\begin{array}{l}\text { rms envelope fitting } \\
\text { along the LEBT }\end{array}$ \\
\hline $\begin{array}{l}\sqrt{\sigma_{11} \sigma_{22}-\sigma_{12}^{2}} \\
\operatorname{mm~mrad}\end{array}$ & 17.2 & 19.3 & 20.4 \\
\hline $\begin{array}{l}\frac{\sigma_{11}}{\sqrt{\sigma_{11} \sigma_{22}-\sigma_{12}^{2}}} \\
\mathrm{~m} / \mathrm{rad}\end{array}$ & 0.16 & 0.21 & 0.23 \\
\hline$-\frac{\sigma_{12}}{\sqrt{\sigma_{11} \sigma_{22}-\sigma_{12}^{2}}}$ & 0.11 & 0.40 & 0.76 \\
\hline $\begin{array}{l}\sqrt{\sigma_{33} \sigma_{44}-\sigma_{34}^{2}} \\
\mathrm{~mm} \operatorname{mrad}\end{array}$ & 17.4 & 16.5 & 22.2 \\
\hline$\frac{\sigma_{33}}{\sqrt{\sigma_{33} \sigma_{44}-\sigma_{34}^{2}}}, \mathrm{~m} / \mathrm{rad}$ & 2.6 & 3.3 & 1.9 \\
\hline$-\frac{\sigma_{34}}{\sqrt{\sigma_{33} \sigma_{44}-\sigma_{34}^{2}}}$ & 0.53 & 0.60 & 1.04 \\
\hline$\sigma_{13}, \mathrm{~mm}^{2}$ & $\cdots$ & $\cdots$ & 6.3 \\
\hline$\sigma_{23}, \mathrm{~mm} \mathrm{mrad}$ & $\ldots$ & $\ldots$ & -62.8 \\
\hline$\sigma_{14}, \mathrm{~mm} \mathrm{mrad}$ & $\cdots$ & $\cdots$ & -2.4 \\
\hline$\sigma_{24}, \operatorname{mrad}^{2}$ & $\cdots$ & $\cdots$ & 12.7 \\
\hline
\end{tabular}

locations. Our image viewers are based on $\mathrm{KBr}$ phosphor screens and a 16-bit monochrome CCD camera ("The Imaging Source", DMK 33GX174 [9]). Both measurements and simulations show a hollow beam cross section in several locations. The hollow structure is induced by the contribution of space charge forces generated due to the different locations of the focal planes along the longitudinal coordinates as a function of the charge-to-mass ratio of an individual ion beam component, as well as by the spherical aberrations of extraction optics and solenoids [7,10]. For moderate beam intensities, up to $\sim 100 \mu \mathrm{A}$, the hollow beam structures shown in the left and middle images in Fig. 5 can be avoided by utilizing weaker focusing solenoids upstream of the first dipole magnet.

Another set of plots, in Fig. 6, shows the measured and simulated beam profiles along the vertical section of the LEBT. The measured and simulated rms beam sizes are consistent to within $\pm 10 \%$. Overall, the TRACK code accurately reproduces the beam rms dimensions and the 2D particle distribution in real space. However, the details of the measured profiles and beam density distributions 

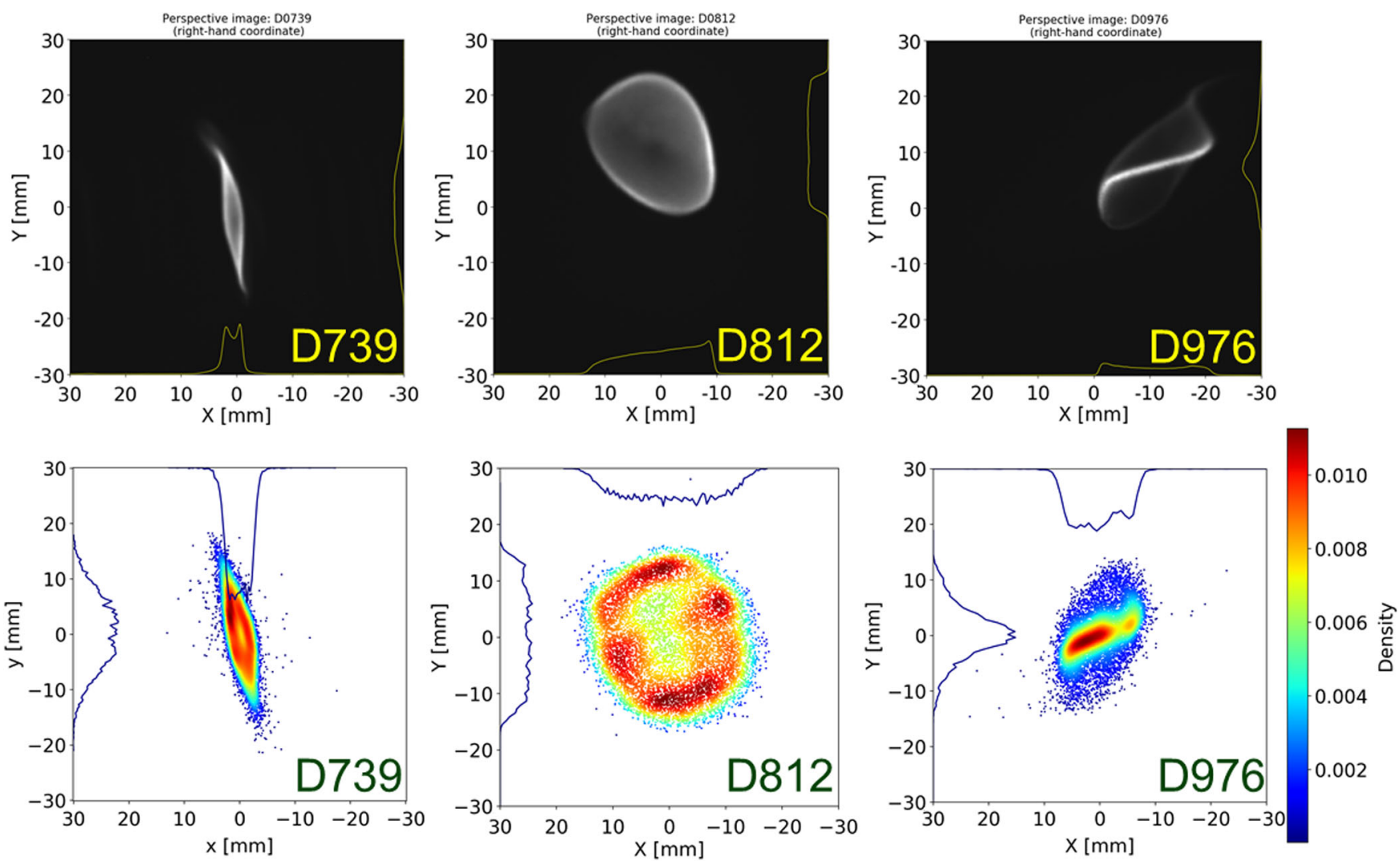

FIG. 5. Measured (top) and simulated (bottom) ${ }^{40} \mathrm{Ar}^{9+}$ beam images along the LEBT. The "D" numbers correspond to the decimeter location of image viewers along the LEBT.

PM_D0856
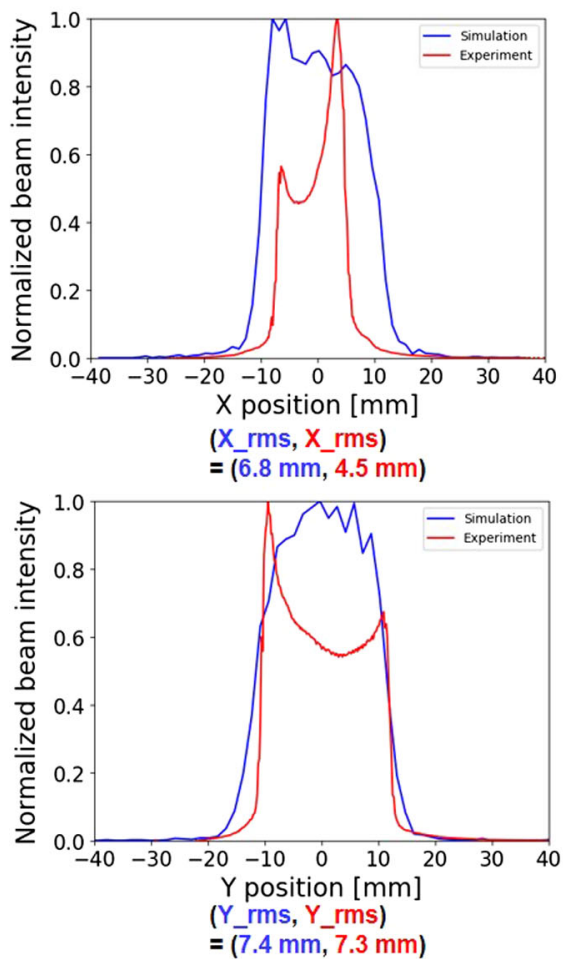

PM_D0885
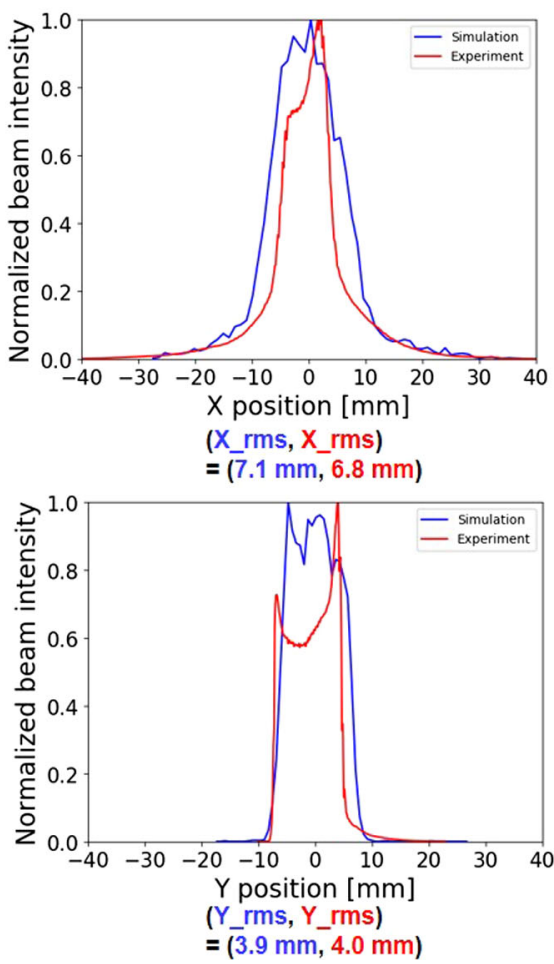

PM_D0912
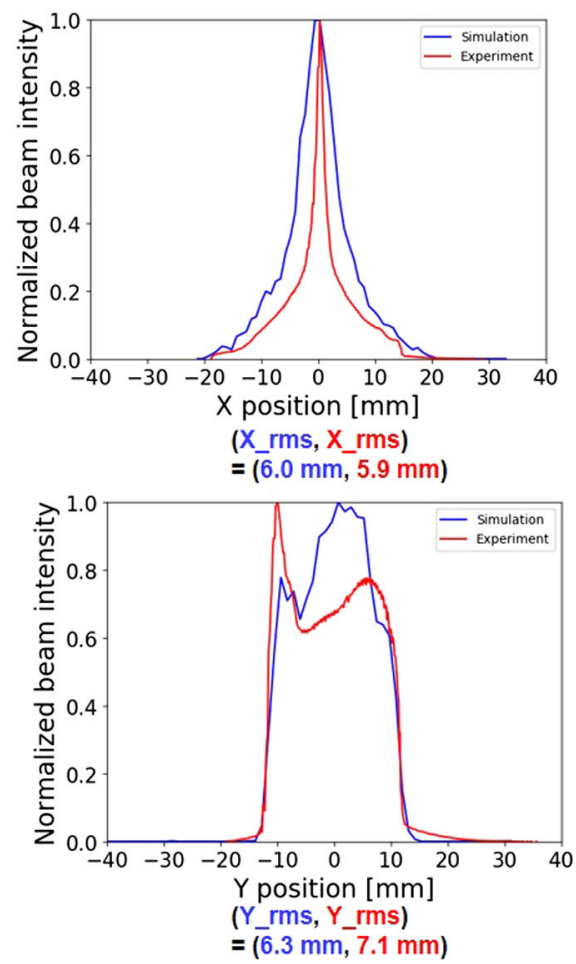

FIG. 6. Measured (red) and simulated (blue) ${ }^{40} \mathrm{Ar}^{9+}$ beam profiles along the LEBT. 


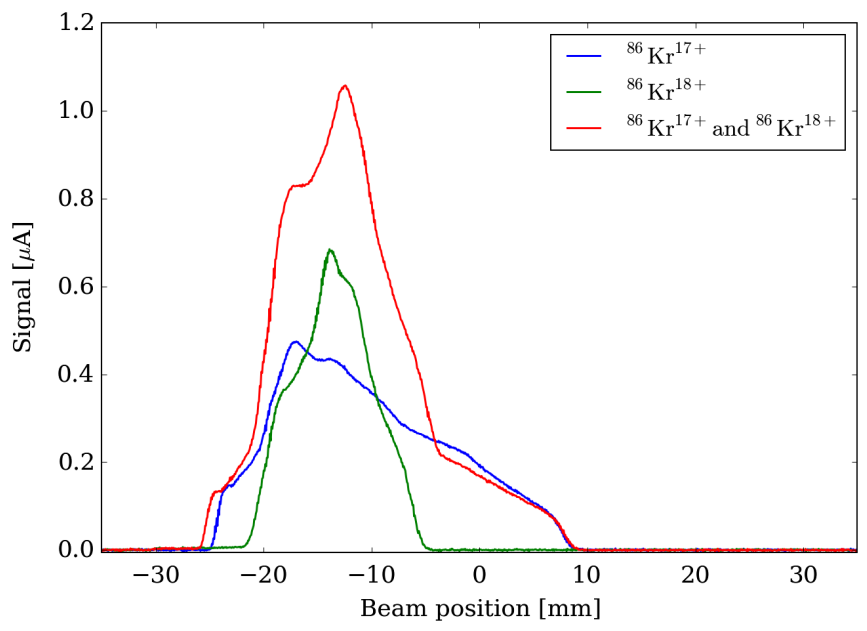

FIG. 7. Beam profiles of ${ }^{86} \mathrm{Kr}^{17+}$ and ${ }^{86} \mathrm{Kr}^{18+}$ measured individually (blue and green) and together (red) upstream of the RFQ.

differ from the simulations, which is most likely related to a simplified initial beam distribution in the $4 \mathrm{D}$ phase space. Due to the complexity of physical processes, the existing computational models of an ECRIS are based on various simplifications and use some empirical parameters to reproduce experimental data. Therefore, we do not have a good computer model to generate the initial multicomponent ion beam distribution.

As was mentioned above, the LEBT is designed to transport dual-charge-state heavy ion beams. To test this feature, we selected dual-charge-state krypton beam, ${ }^{86} \mathrm{Kr}^{17+}$ and ${ }^{86} \mathrm{Kr}^{18+}$ from the ion source and transported it in the LEBT. The settings of all beam optics devices were scaled with the ratio $\frac{86}{17.5} \frac{9}{40}=1.106$ with respect to the setting for the ${ }^{40} \mathrm{Ar}^{9+}$ beam. The dual-charge-state krypton beam was transported with nearly $100 \%$ efficiency to the entrance of the RFQ. The intensities of ${ }^{86} \mathrm{Kr}^{17+}$ and ${ }^{86} \mathrm{Kr}^{18+}$ were 33 and $27 \mu \mathrm{A}$, respectively. Figure 7 shows the transverse profiles of single- and dual-charge-state krypton beam at the entrance of the RFQ. The dual-charge-state beam is well combined prior to injection into the RFQ. These measurements suggest that we can nearly double the intensity of heavy ion beams in the FRIB linac by acceleration of dual-charge-state ion beams. To implement this feature, we still need to install the velocity equalizer upstream of the RFQ [11].

\section{BEAM ACCELERATION IN THE RFQ}

The FRIB RFQ operates at $80.5 \mathrm{MHz}$ and was designed for an initial synchronous phase of $-35^{\circ}$. A multiharmonic rf buncher (MHB) with the fundamental frequency of 40.25 MHz provides a bunched beam to the entrance of the RFQ with a small longitudinal emittance, while the relatively small RFQ acceptance serves as a filter of the beam longitudinal phase space and eliminates halo particles. In addition, the MHB in combination with the

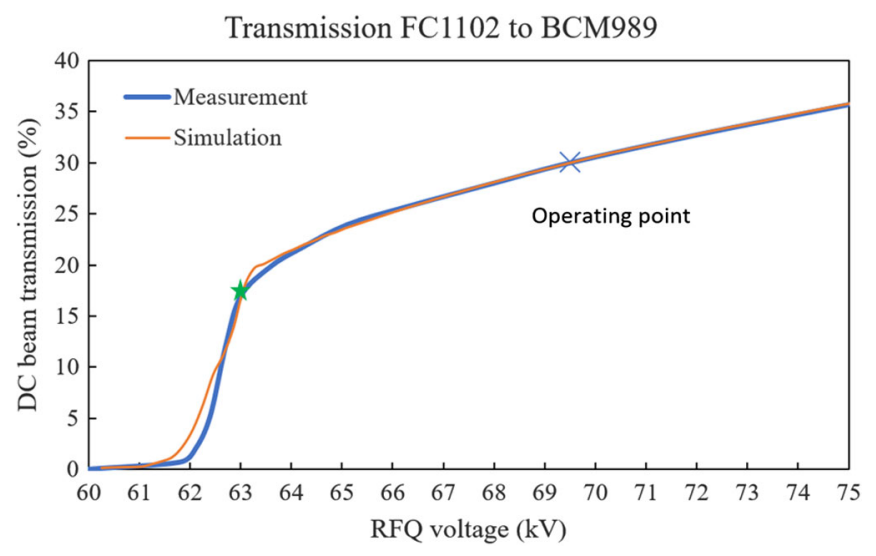

FIG. 8. Measured (red) and simulated (blue) RFQ acceleration efficiency as a function of the vane voltage. The star shows the transmission at the RFQ threshold voltage.

velocity equalizer [11] provides the possibility to inject a dual-charge-state heavy ion beam into the RFQ. The velocity equalizer [12] has not yet been installed. Our commissioning studies in the RFQ and LS1 have dealt with only a single charge state ion beam.

The first step in the RFQ tuning is the measurement of the dc beam transmission efficiency as a function of the vane voltage. The latter varies along the RFQ, and we refer to the highest intervane voltage which is realized at the high energy end of the RFQ. There are two FCs in the MEBT, as shown in Fig. 2: on the straight line with the RFQ and behind the $45^{\circ}$ bending magnet. If particles' energy is below $100 \mathrm{keV} / \mathrm{u}$, they do not propagate to the FC located on the straight line. Therefore, the plot in Fig. 8 shows the acceleration efficiency of the RFQ. The RFQ itself transmits almost all injected particles, accelerated and unaccelerated. The unaccelerated portion of the beam is lost in the focusing quadrupoles located between the RFQ and FC. Detailed 3D models of the MHB and RFQ were created in the TRACK code to support beam dynamics studies in the RFQ [13]. The results of beam transmission simulations are shown in Fig. 8, together with the measured data.

The designed synchronous phase in the acceleration section of the RFQ is $\varphi_{s}=-25^{\circ}[14,15]$. Therefore, if the RFQ voltage is below the threshold voltage [16] $V_{\text {th }}=$ $V_{0} \cos \varphi_{s}=63 \mathrm{kV}$, where $V_{0}=69.5 \mathrm{kV}$ is the design voltage for ${ }^{40} \mathrm{Ar}^{9+}$ beam, there is no acceleration to the design energy of $0.5 \mathrm{MeV} / \mathrm{u}$. The threshold voltage is marked in Fig. 8 with a star.

Figure 9 shows the acceptance of the RFQ and the beam phase space plots with the setting of the MHB for (a) maximum transmission and (b) minimum longitudinal emittance in the RFQ. The measured acceleration efficiency of the RFQ (with the MHB set for maximum transmission) is close to the simulated value of $84 \%$. The MHB can form very small longitudinal emittance with a slightly lower transmission compared to the maximum value. 

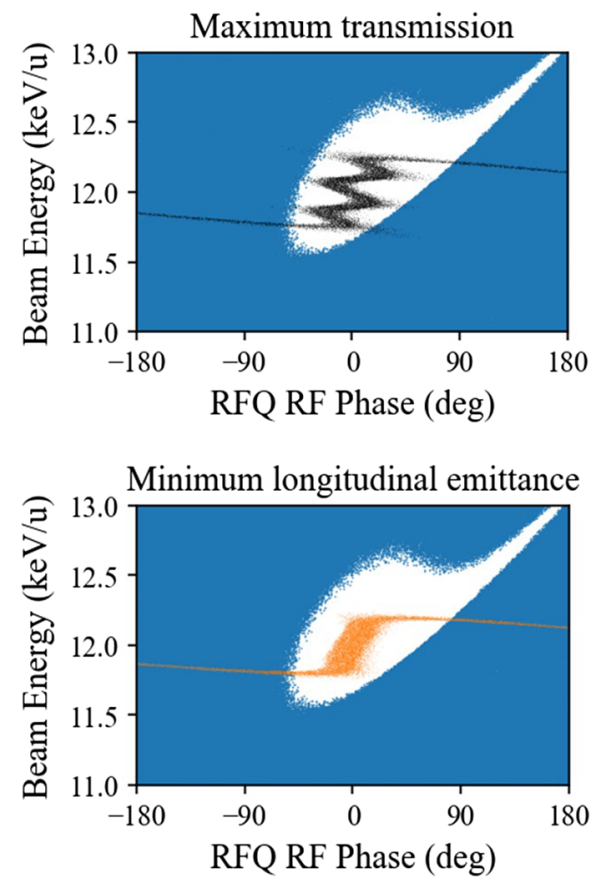

FIG. 9. RFQ acceptance and the bunch phase space images formed with MHB in two modes: the MHB is tuned to maximize the acceleration efficiency (a) and to minimize the longitudinal emittance (b).

The RFQ exit beam energy, $0.5 \mathrm{MeV} / \mathrm{u}$, was verified using the $45^{\circ}$ dipole magnet and time-of-flight (TOF) measurements using beam induced phase signals in the BPMs. The rms transverse beam emittance in the MEBT was reconstructed using the beam rms size measured with a profile monitor while the upstream quadrupole field was varied. The profile monitor devices consist of actuated horizontal, vertical and $45^{\circ}$-angled wires. These measurements are taken at different quadrupole fields, but with $100 \%$ beam transmission to the FC located behind the profile monitor and quadrupole triplet. Typical beam sizes

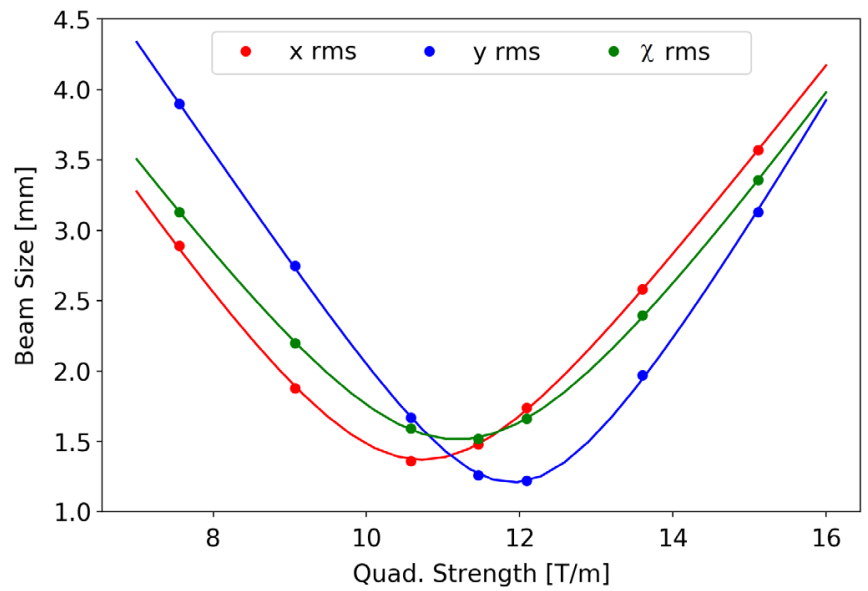

FIG. 10. Beam rms sizes as a function of the quadrupole strength.

and XY-coupling term, $\chi$, as a function of the quadrupole gradient are plotted in Fig. 10. All ten beam rms parameters in the $\sigma$ matrix, $\sigma_{11}, \sigma_{12}, \sigma_{13}, \sigma_{14}, \sigma_{22}, \sigma_{23}, \sigma_{24}, \sigma_{33}, \sigma_{34}, \sigma_{44}$, can be found as a result of fitting using the FLAME code. Detailed analysis of MEBT beam transport revealed that the overlapping of magnetic quadrupole fields in the triplets significantly affects the value of $\sigma$-matrix elements. The fitting code FLAME was modified to include overlapping focusing fields in triplets. The overlapping of the quadrupole fields did not introduce noticeable correlation in the quadrupole current settings, due to the relatively small beam size with respect to the aperture. The full size of the beam occupies less than $50 \%$ of the aperture. For the same reason, we do not observe noticeable coupling between the $\mathrm{X}$ and $\mathrm{Y}$ motion of ions.

Figure 11 shows the designed and measured rms phase space ellipses of the beam exiting the RFQ. The mismatch factor [17] between simulated and measured Twiss parameters varies from day to day, due to ECR stability from cold start every day. Average mismatch was $14 \%$ and $27 \%$ in the
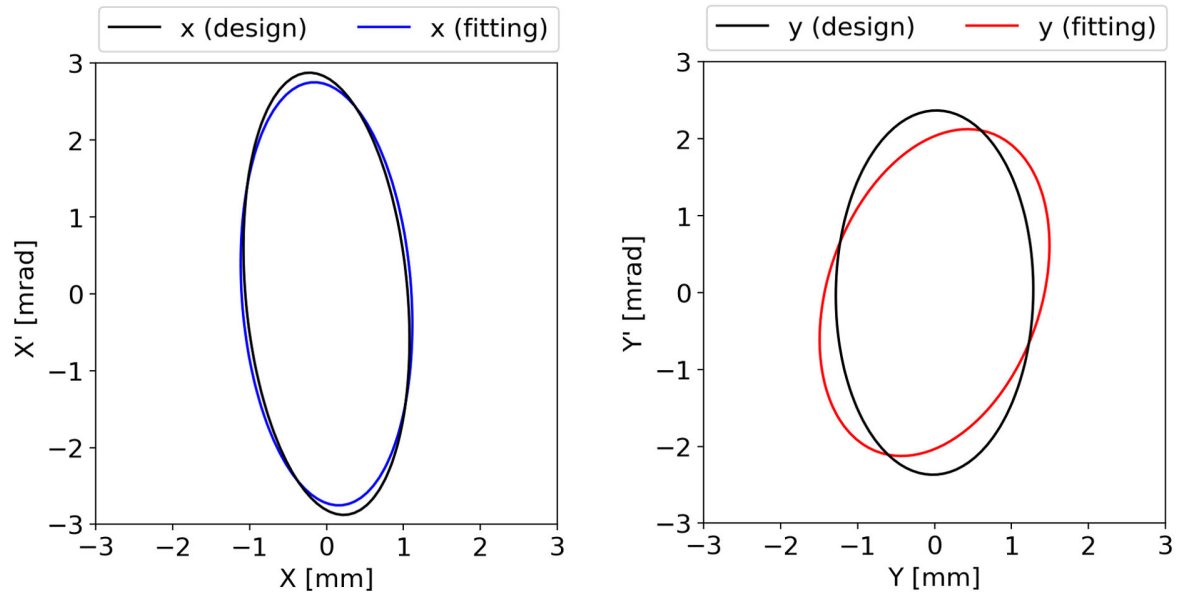

FIG. 11. Designed and measured rms phase space ellipses of the beam exiting the RFQ. 
horizontal and vertical planes respectively. The beam dynamics simulations for the RFQ show that the beam Twiss parameters at the RFQ exit are sensitive to the beam matching into the RFQ. The daily changes of beam Twiss parameters are related to the current operational mode of the front end, which does not yet operate 24/7 and requires cold startup every day.

The beam normalized rms emittances, in both $\mathrm{XX}^{\prime}$ and $\mathrm{YY}^{\prime}$ planes, are equal to $(0.1 \pm 0.01) \pi \mathrm{mm} \operatorname{mrad}$. During early MEBT commissioning, we measured $\sim 0.25 \pi \mathrm{mm} \mathrm{mrad}$ for both the horizontal and vertical planes. It took some time to determine the source of the emittance growth in the RFQ. We found that if the center of the beam entering the RFQ was tuned for maximum transmission of accelerated particles only, it may result in an emittance growth due to the misaligned beam entering the RFQ. The lowest value of beam emittance in the MEBT was provided if the incoming beam was centered by maximizing the transmission of both accelerated and unaccelerated particles in the RFQ, which was done using the BCM located just after the RFQ.

\section{ARGON AND KRYPTON BEAM ACCELERATION TO $2.3 \mathrm{MEV} / \mathrm{U}$}

To characterize beams accelerated in the first three cryomodules, we have developed, built and installed a temporary diagnostics beam line (D-station). Major components of the D-station are shown in Fig. 12. There are 15 total BPMs; in the MEBT (four), inside the cryomodules (six), between the cryomodules (two) and D-station (three). The beam induced rf signal is available from all BPMs. All BPMs were calibrated to support absolute beam velocity measurements, using the TOF technique and any pair of BPMs. There are three halo monitor rings (HMRs) located in the warm section between the cryomodules. The D-station included a silicon detector [18], which was used to measure both the absolute energy and bunch length.

Prior to beam commissioning, all SC cavities were cooled down to $4.5 \mathrm{~K}$ and conditioned at accelerating gradients exceeding the design value of $5.1 \mathrm{MV} / \mathrm{m}$ by $10 \%$. This design gradient provides $0.81 \mathrm{MV}$ accelerating voltage for the beam entering the cavity with the optimal velocity. The phase scan procedure was applied to determine synchronous phases for each MEBT buncher and each SC cavity. This procedure constitutes the measurement of the beam induced signal in a downstream BPM as a function of cavity rf field phase. A typical cavity phase scan curve is illustrated in Fig. 13. This procedure was applied at $\sim 1 \mathrm{MV} / \mathrm{m}$ accelerating gradient to avoid a transverse steering of the beam, which strongly depends on the rf field phase. The cavity synchronous phase was set to the design value, which is typically equal to $-30^{\circ}$ from peak acceleration, as shown in Fig. 13. The cavity accelerating gradient was calibrated by measuring the absolute beam energy. We typically used three BPMs, paired into two sets, for robust TOF measurements. The BPM signal amplifiers have very high sensitivity, therefore stable beam phase and position can be obtained for $\sim 40 \mathrm{nA}$ beam current. The accuracy of absolute beam energy measurements is high, typically $\sim 20 \mathrm{keV} / \mathrm{u}$, and can be easily improved by selecting BPM pairs with a longer distance between the BPMs. The uncertainty of the beam energy due to the large phase advance between the BPMs, which can include multiple $360^{\circ}$ periods, was not a concern because the beam

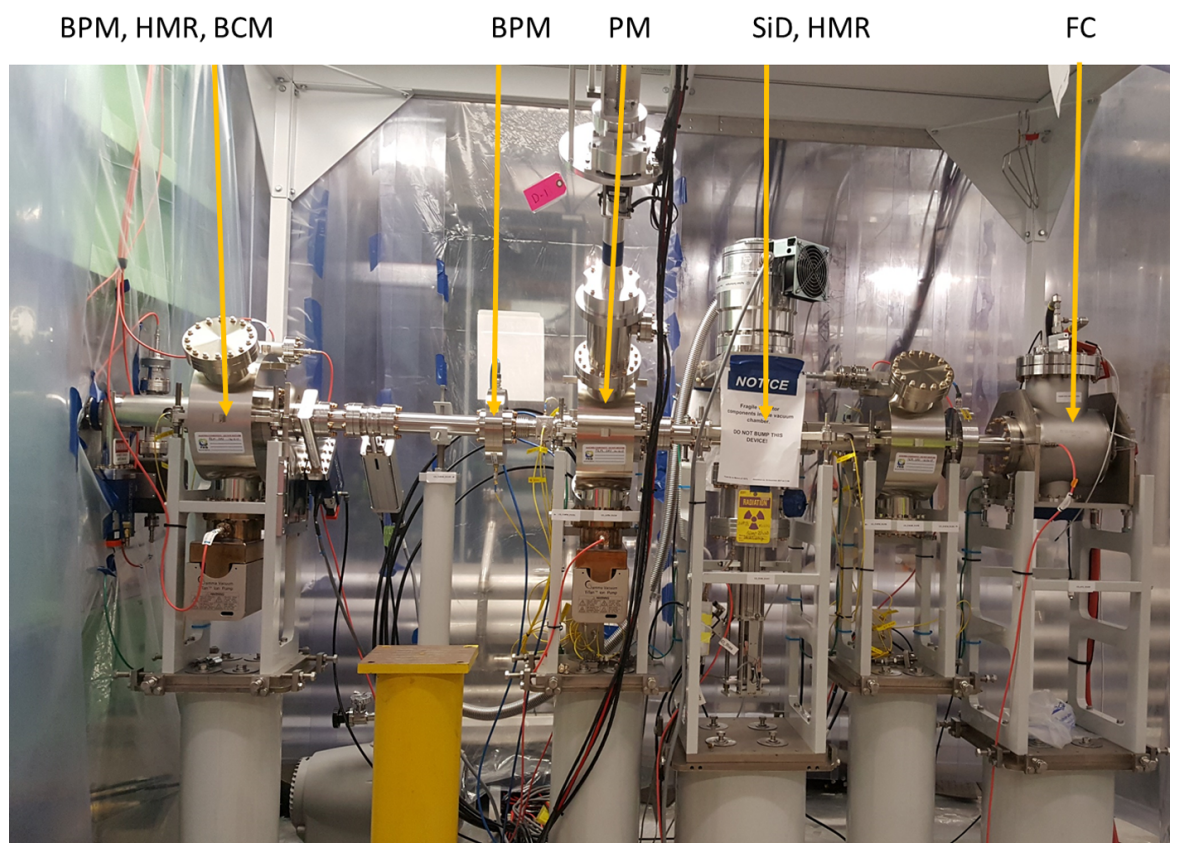

FIG. 12. Temporary diagnostics station (D-station) located downstream of the third cryomodule. 


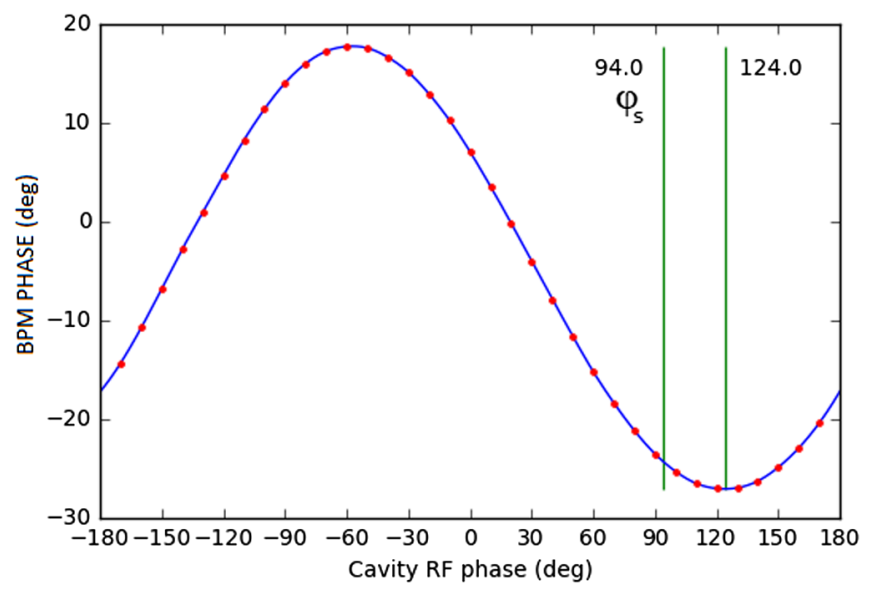

FIG. 13. Phase scan signature.

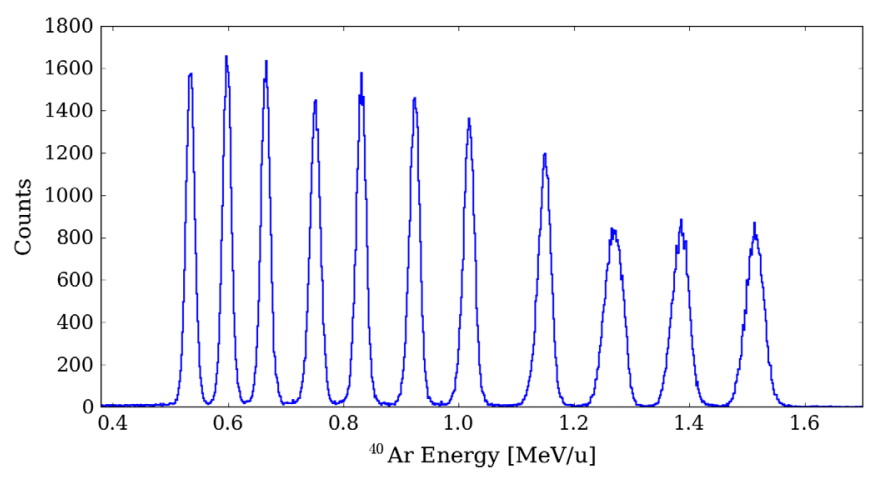

FIG. 14. Absolute beam energy measured with silicon detector after each of 11 SC cavities.

energy after the RFQ was measured independently with the $45^{\circ}$ bending magnet. Utilizing three BPMs is another method to reduce TOF phase uncertainty. In this manner, the beam energy is known with high accuracy upstream of the SC cavity which is being set. In addition, the beam energy was measured after each SC cavity with the silicon detector, as depicted in Fig. 14. In the beginning of the beam commissioning, one SC cavity was disabled to satisfy radiation safety requirements. Argon beam was accelerated to $2.01 \mathrm{MeV} / \mathrm{u}$ using $11 \mathrm{SC}$ cavities. Krypton beam was accelerated to the same energy by simply scaling all accelerating and focusing fields. The designed energy in the first three cryomodules of FRIB was selected to be $1.46 \mathrm{MeV} / \mathrm{u}$ for all ion species, to minimize longitudinal emittance growth due to rapid acceleration while beam velocity is low [12].

After beam acceleration in the first three cryomodules was demonstrated and major project milestones were met, the argon beam was accelerated to $2.3 \mathrm{MeV} / \mathrm{u}$ using all 12 cavities at the designed level of accelerating gradients. SC solenoids provide focusing in the cryomodules. The MEBT quadrupoles were tuned to match the argon beam to the acceptance of the solenoidal focusing channel, which were set to provide a $\sim 60^{\circ}$ phase advance of transverse oscillations over a period of the focusing channel. Only very small adjustments of several steering dipole magnets were required to align the accelerated beam within $\pm 1.5 \mathrm{~mm}$ in all BPMs, as illustrated in Fig. 15.

After setting the phase and amplitude to the designed values in all SC cavities and transverse beam matching, we decided to evaluate longitudinal rms emittance by using one of the SC cavities as a buncher and varying its field to change the bunch time profile at the location of the silicon detector. The $1.03 \mathrm{MeV} / \mathrm{u}$ beam time profile (bunch length) was measured with the silicon detector. Figure 16 shows the results of these measurements for two settings of the MHB: (1) for maximum transmission and (2) for minimum of longitudinal emittance. The shortest rms bunch width for 1.27 MeV/u beam was measured at $128 \mathrm{ps}\left(3.7^{\circ}\right.$ at $80.5 \mathrm{MHz}$ ), as illustrated in Fig. 17. It should be noted that $100 \%$ of particles are within $\sim 30^{\circ}$ of the full bunch width.

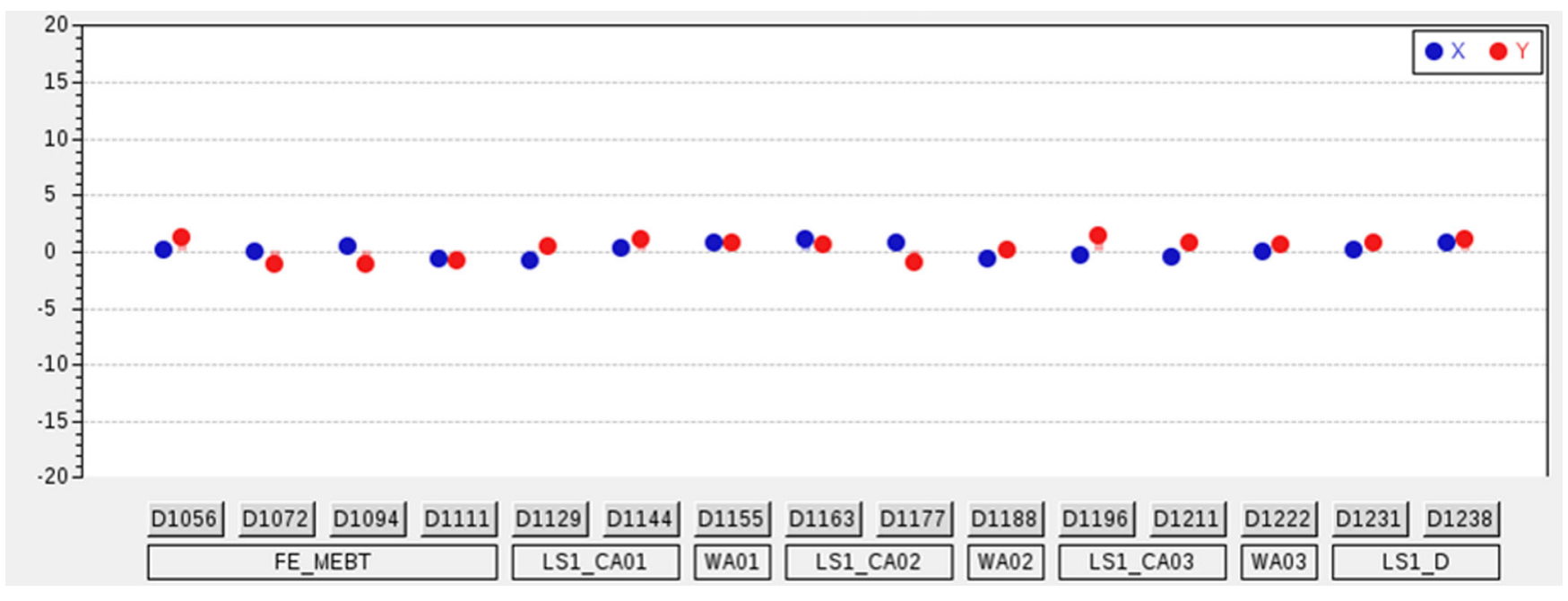

FIG. 15. Argon beam position in BPMs along the MEBT, cryomodules and D-station. 


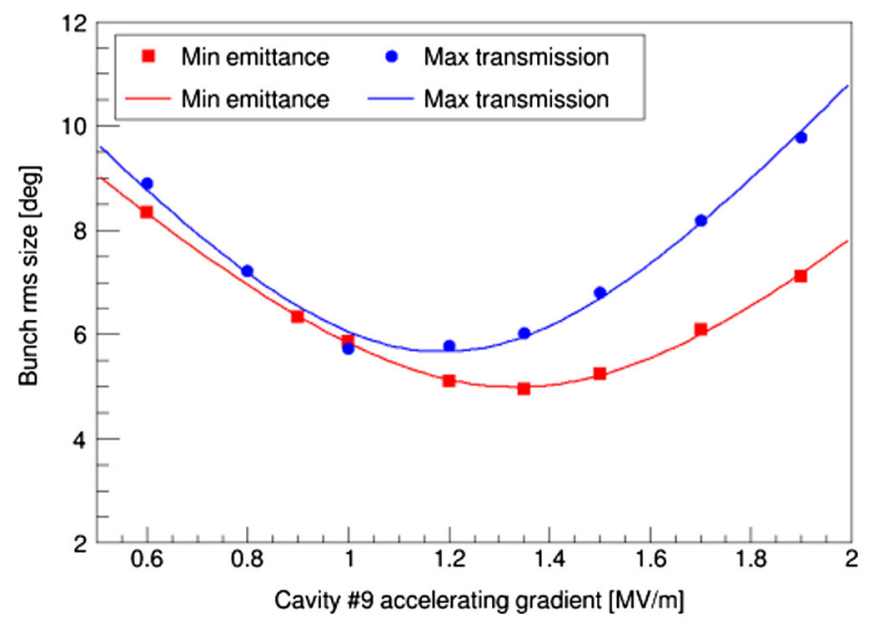

FIG. 16. Beam longitudinal $\mathrm{rms}$ size as a function of the cavity accelerating gradient for two cases of the MHB tuning: (1) maximum transmission (blue) and (2) minimum longitudinal emittance (red).

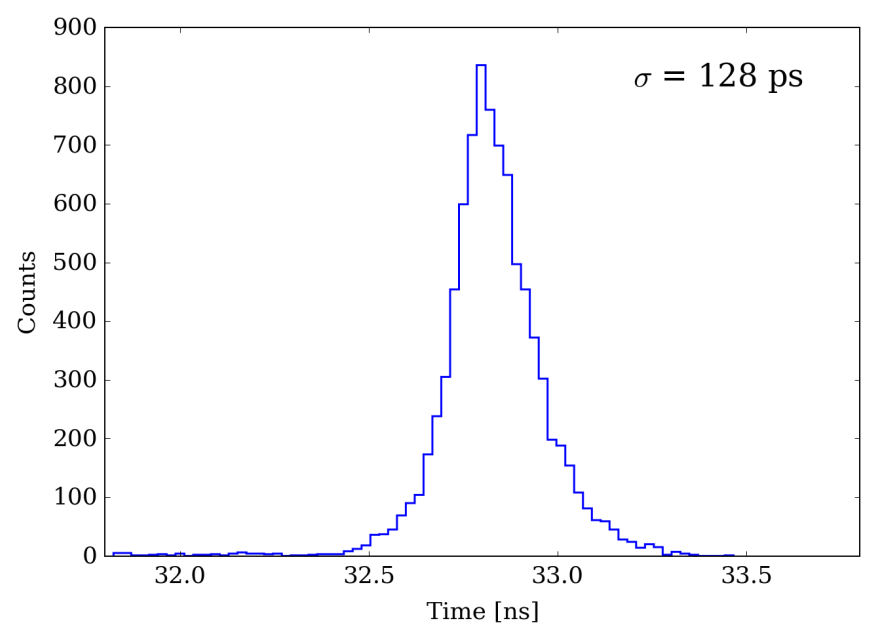

FIG. 17. Bunch time profile measured with the silicon detector.

This confirms that the longitudinal emittance formed with the $\mathrm{MHB}$, and filtered with the RFQ, is halo-free at the relative level of $5 \times 10^{-5}$ based on the number of counts in the silicon detector. The longitudinal rms emittances for both settings of the MHB are given in Table II.

In order to evaluate the transverse rms emittance of the accelerated beam, the strength of the last SC solenoid was varied in the third cryomodule and the beam profiles were

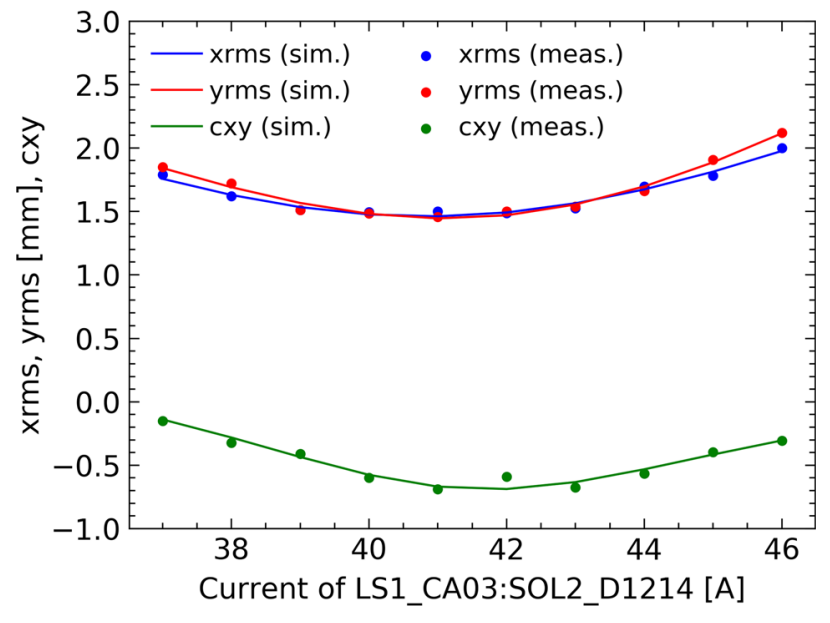

FIG. 18. Beam rms size and XY coupling term as a function of the solenoid current, cxy is the coupling coefficient.

measured with the three-wire PM located in the D-station. The results of these measurements are shown in Fig. 18. According to these measurements, there is $\sim 20 \% \mathrm{rms}$ emittance growth from the MEBT to the D-station. Detailed analysis of the beam dynamics in 3D fields shows that during these measurements the transverse beam envelope matching into the cryomodule section was not perfect and did not include the effect of realistic fields in the triplets.

The measured beam parameters, both in the transverse and longitudinal phase space, are very close to the designed values. The beam is well prepared for the following stages of beam commissioning of the FRIB driver linac.

After the completion of beam studies, we accelerated $33 \mu \mathrm{A}$ argon beam to the designed $1.46 \mathrm{MeV} / \mathrm{u}$ beam energy through the first three cryomodules. A pulsed beam with $100 \mathrm{~Hz}$ repetition rate was formed by the electrostatic chopper located in the LEBT. We used a commercial Faraday cup as a beam absorber, model FC58 built by National Electrostatic Corporation [19], located at the end of the D-station, $2.3 \mathrm{~m}$ downstream of the cryomodules. The beam duty cycle was gradually increased from $1 \%$ to $30 \%$ while we watched the residual vacuum pressure in the cryomodules and near the FC. Due to the intense outgassing of the FC and slight increase of the pressure from $1 \times 10^{-9}$ to $2 \times 10^{-9}$ Torr in the third cryomodule, we decided not to pursue higher duty cycle. During this experiment, we did not observe any signal above the noise level in the HMRs located between the cryomodules and in

TABLE II. Longitudinal rms emittance of accelerated argon beam.

\begin{tabular}{lccc}
\hline \hline MHB setting & Transmission $(\%)$ & $\begin{array}{c}\text { Measured rms emittance } \\
(\pi \mathrm{keV} / \mathrm{u} \mathrm{nsec})\end{array}$ & $\begin{array}{c}\text { Simulated rms emittance } \\
(\pi \mathrm{keV} / \mathrm{u} \mathrm{nsec})\end{array}$ \\
\hline Maximum transmission & 84 & 0.19 & 0.14 \\
Minimum emittance & 76 & 0.14 & 0.12 \\
\hline \hline
\end{tabular}




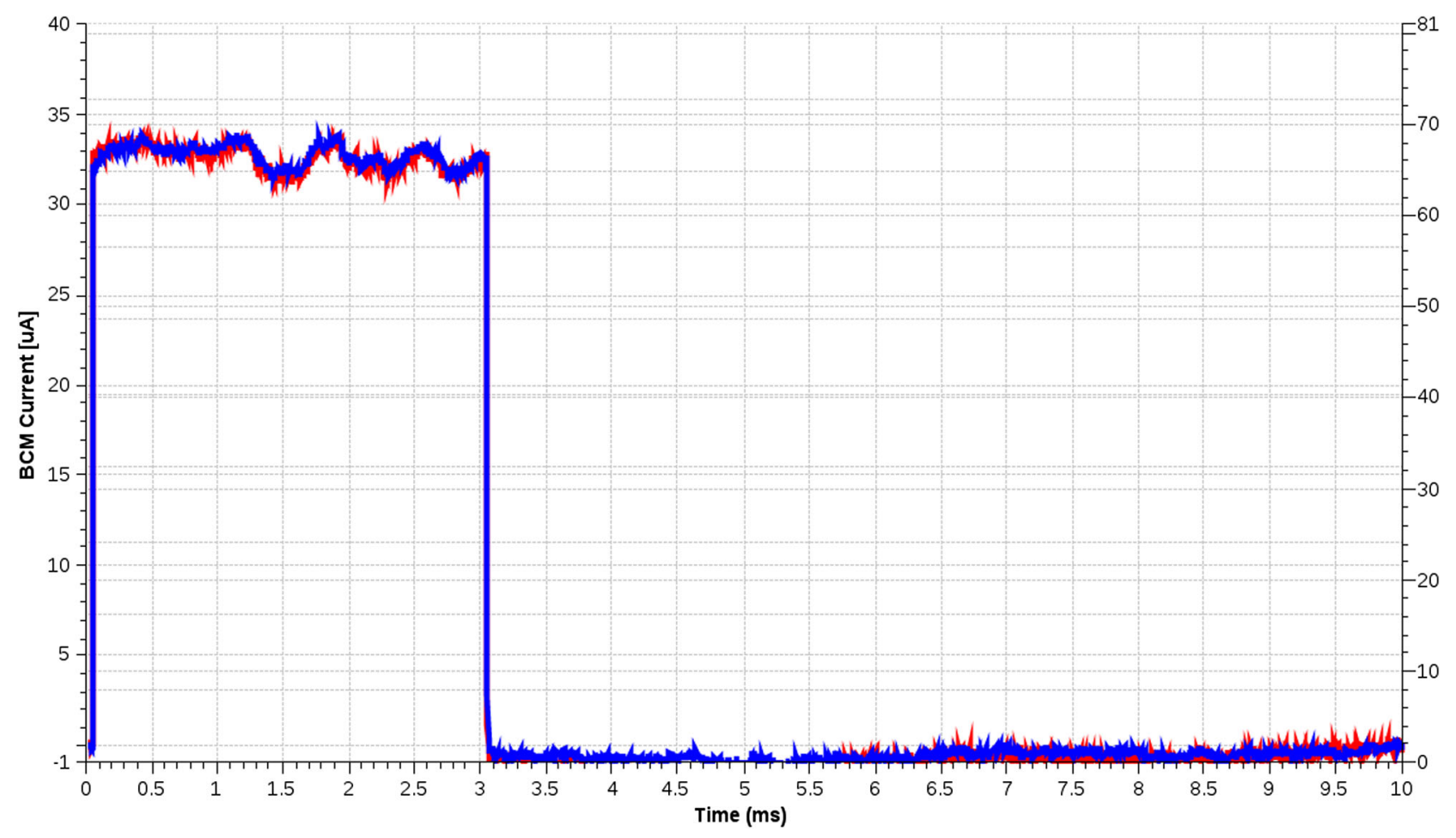

FIG. 19. Beam peak current upstream (red) and downstream (blue) of the first three cryomodules. Pulse length is $3 \mathrm{~ms}$ at $100 \mathrm{~Hz}$ repetition rate.

the D-station, despite the very high sensitivity of the HMRs which is $\sim 0.5 \mathrm{pA}$ and corresponds to $\sim 2 \times 10^{-5}$ relative level of beam losses. If $33 \mu \mathrm{A} \mathrm{cw}$ argon beam was accelerated to the full designed energy of $285 \mathrm{MeV} / \mathrm{u}$ in the completed FRIB linac, it would correspond to $\sim 40 \mathrm{~kW}$ beam power on the target. Figure 19 illustrates the signals from two BCMs located in the MEBT (red) and D-station (blue). The BCM signals are fully overlapped, indicating no losses. The machine protection system was activated to shut off the ECRIS if the differential signal from these two BCMs exceeded $0.6 \mu \mathrm{A}$.

\section{SUMMARY}

The FRIB front end and first three cryomodules were successfully commissioned with beam. The initial study of the beam parameters demonstrated very good consistency with the design parameters. After the appropriate setting of MHB, RFQ, MEBT and accelerating and focusing fields in the first three cryomodules, the beam acceleration in the cryomodules did not show any beam losses and allowed us to demonstrate high power equivalent beam in the pulsed mode at 30\% duty cycle. Further increase of beam power was limited due to intense outgassing of the Faraday cup located in close vicinity to the cryomodule. Very little beam steering correction was required to minimize the beam center deviation in all 15 BPMs to within $\pm 1.5 \mathrm{~mm}$. These studies demonstrated high alignment accuracy of all SC components. All accelerator hardware showed very reliable operation within the design parameters space.

\section{ACKNOWLEDGMENTS}

The authors greatly appreciate contributions of all FRIB staff to the successful operation of the front end and the first three cryomodules. This work was supported by the U.S. Department of Energy Office of Science under Cooperative Agreement No. DE-SC0000661 and the National Science Foundation under Cooperative Agreement No. PHY1102511, the State of Michigan and Michigan State University.

[1] E. Pozdeyev et al., FRIB front end construction and commissioning, IPAC-18, Vancouver, BC, Canada, p. 58, http://accelconf.web.cern.ch/AccelConf/ipac2018/ papers/mozgbf1.pdf.

[2] G. A. Machicoane, ARTEMIS-B: A room-temperature test electron cyclotron resonance ion source for the National Superconducting Cyclotron Laboratory at Michigan State University, Rev. Sci. Instrum. 77, 03A322 (2006).

[3] G. Machicoane et al., Design status of ECR ion source and LEBT for FRIB, ECRIS'12, p. 182, http://accelconf.web .cern.ch/AccelConf/ECRIS2012/papers/thyo03.pdf.

[4] H. Ren, E. Pozdeyev, S. M. Lund, G. Machicoane, X. $\mathrm{Wu}$, and G. Morgan, Beam simulation studies of ECR beam extraction and low energy beam transport for FRIB, 
Rev. Sci. Instrum. 87, 02B919 (2016), https://doi.org/10 $.1063 / 1.4934621$.

[5] P. N. Ostroumov, V. N. Aseev, and B. Mustapha, TRACKA code for beam dynamics simulations in accelerators and transport lines with 3D electric and magnetic fields, https:// www.phy.anl.gov/atlas/TRACK/Trackv39/Manuals/tv39_ man_index.html.

[6] Z. He, Y. Zhang, J. Wei, Z. Liu, and R. M. Talman, Linear envelope model for multi-charge state linac, Phys. Rev. ST Accel. Beams 17, 034001 (2014).

[7] T. Yoshimoto et al., Ion beam studies in the FRIB front end, IPAC'18, Vancouver, BC, Canada, p. 1094, http://accelconf .web.cern.ch/AccelConf/ipac2018/papers/tupal040.pdf.

[8] J. A. Nelder and R. Mead A simplex method for function minimization, Computer Journal (UK) 7, 308 (1965).

[9] https://www.theimagingsource.com/.

[10] M. Reiser, Theory and Design of Charged Particle Beams (Wiley, New York, 1994).

[11] P. N. Ostroumov et al., Heavy-ion beam acceleration of two-charge states from and ECR ion source, LINAC'00, Monterey, CA, http://accelconf.web.cern.ch/AccelConf/ 100/papers/MOD01.pdf.
[12] Q. Zhao et al., FRIB accelerator beam dynamics design and challenges, HB'12, p. 404, https://accelconf.web.cern .ch/accelconf/HB2012/papers/weo3b01.pdf.

[13] A. S. Plastun et al., Longitudinal beam dynamics in FRIB and ReA linacs, 13th International Computational Accelerator Physics Conference ICAP2018, Key West, FL (JACoW, Geneva, Switzerland, 2018), p. 341, http:// icap2018.vrws.de/papers/wepaf04.pdf.

[14] Q. Zhao et al., Design improvement of the RIA $80.5 \mathrm{MHz}$ RFQ, LINAC'04, p. 599, http://accelconf.web.cern.ch/ AccelConf/104/PAPERS/THP03.PDF.

[15] N. Bultman et al., Design of the FRIB RFQ, IPAC'13, p. 2866, http://accelconf.web.cern.ch/AccelConf/IPAC2013/ papers/wepfi075.pdf.

[16] I. M. Kapchinskiy, Theory of Resonance Linear Accelerator (Harwood Academic Publisher, New York, 1985).

[17] K. R. Crandall and D. P. Rusthoi, TRACE 3-D documentation, Report No. LA-UR-97-886, 3rd ed., 1997.

[18] https://www.canberra.com.

[19] http://www.pelletron.com/. 